\title{
Forward to the past
}

\author{
Alessandro Carlini ${ }^{1,2,3 *}$, Rossana Actis-Grosso ${ }^{3}$, Natale Stucchi $^{3}$ and Thierry Pozzo ${ }^{1,2,4,5}$ \\ ${ }^{1}$ UFR-Sciences et Techniques des Activités Physiques et Sportives, Université de Bourgogne, Dijon, France \\ 2 Institut National de la Santé et de la Recherche Médicale, Cognition, Action, and Sensorimotor plasticity, Dijon, France \\ ${ }^{3}$ Department of Psychology, Università degli Studi di Milano-Bicocca, Milan, Italy \\ ${ }^{4}$ Department of Robotics, Brain, and Cognitive Sciences, Italian Institute of Technology, Genoa, Italy \\ ${ }^{5}$ Institut Universitaire de France, Institut National de la Santé et de la Recherche Médicale, Université de Bourgogne, Dijon, France
}

\section{Edited by:}

John J. Foxe, Albert Einstein College

of Medicine, USA

\section{Reviewed by:}

Dirk Kerzel, Université de Genève, Switzerland

Yoshikazu Ugawa, Fukushima

Medical Universtiy, Japan

${ }^{*}$ Correspondence:

Alessandro Carlini, Institut National de la Santé et de la Recherche Médicale U1093, Université de Bourgogne, Campus Universitaire, BP 27877, Dijon 21078, France.

e-mail: alessandro.carlini@ u-bourgogne.fr
Our daily experience shows that the CNS is a highly efficient machine to predict the effect of actions into the future; are we so efficient also in reconstructing the past of an action? Previous studies demonstrated we are more effective in extrapolating the final position of a stimulus moving according to biological kinematic laws. Here we address the complementary question: are we more effective in extrapolating the starting position (SP) of a motion following a biological velocity profile? We presented a dot moving upward and corresponding to vertical arm movements that were masked in the first part of the trajectory. The stimulus could either move according to biological or non-biological kinematic laws of motion. Results show a better efficacy in reconstructing the SP of a natural motion: participants demonstrate to reconstruct coherently only the SP of the biological condition. When the motion violates the biological kinematic law, responses are scattered and show a tendency toward larger errors. Instead, in a control experiment where the full motions were displayed, no-difference between biological and non-biological motions is found. Results are discussed in light of potential mechanisms involved in visual inference. We propose that as soon as the target appears the cortical motor area would generate an internal representation of reaching movement. When the visual input and the stored kinematic template match, the SP is traced back on the basis of this memory template, making more effective the SP reconstruction.

Keywords: motion inference, kinematics, internal models, visual perception

\section{INTRODUCTION}

Our daily experience shows that the CNS is a highly efficient machine to predict the effect of actions. This capability to project into the future is an essential ability to survive (e.g., to capture a prey, or avoid to be caught by a predator). Indeed, efficient ability to interact with the environment strongly depends on the possibility to compensate for the delay generated by the elaboration process and signal transmission by the nervous system (Nijhawan, 1994). In this frame, the use of internal models that predict the sensory and the motor effect of action execution is essential to prepare the motor plan (Jordan and Rumelhart, 1992; Miall and Wolpert, 1996; Wolpert and Kawato, 1998; Kilner et al., 2007).

The ending position of a movement is crucial for error computation, feedback regulation, and tuning the sensory-motor predictions. In contrast, the starting point of an action is a past input no more available at the end of the action; consequently it is not verified or adjusted like the ending position.

Another illustration of the apparent lack of dynamic process similar to action prediction but reversing the normal direction of action, is our limited ability to replay an action in a reversal order (i.e., rewind, from the end to the beginning) a task that requires an important cognitive effort. It's easy to verify how difficult is to produce a reversal action from the end to the beginning, during the hand-writing for example, or to play a sporting gesture as a tennis serve in a reversal order. These observations raise an important question: since the brain appears mainly proactive, continuously projecting itself into the future, is it able to reconstruct the past?

The same question has been answered for the "future" of a movement, with a psychophysical experimental paradigm where subjects were asked to estimate the final position of a moving dot; the dot motion corresponds to the position of a finger of a moving hand during a pointing movement, that was masked in the last part of its trajectory after biological and after non-biological motion display (Pozzo et al., 2006). Both accuracy and precision increased for the estimation of the final position of a biological motion. This result has been interpreted as evidence that motion inference relies on a specific internal model-an actionperception matching system - that contains specific kinematic details, which can be rapidly recalled during motion observation. If in general knowing the past is essential to predict the future, with the biological motion this would not be necessary: even when not available, the past should be known on the basis of a stereotyped kinematic template. Consequently, there should be an improvement also in human ability to recover the starting 
point of a movement with this typical kinematic pattern, whose initial part is partially hidden.

The aim of the present study is to investigate this capacity by testing subjects' performance to estimate the starting position of a movement. More precisely, we test our capacity to infer the starting point (SP) of a dot moving upwards and corresponding to upward arm pointing movements that were masked in the first part of the trajectory. To this aim we displayed to the observers a moving dot reproducing or violating this natural kinematic and asked them to infer the starting point of the trajectory that has been masked.

The use of a single dot reproducing a natural kinematic (a human kinematic, hereafter labeled as "BIO") results from experiment outcomes in human movement recognition; since the works of Johansson (Johansson, 1973) it is known that humans are able to recognize in a consistent manner the movement of a human body, even if it is presented in a rather reduced way, that is, through few or only a single visible markers placed on some relevant points (i.e., joints) of the body.

Subsequent works showed how the perceptual system is well attuned to some peculiarity of human movement kinematics (e.g., the relation between velocity and curvature: Lacquaniti et al., 1983; Viviani and Stucchi, 1989, 1992; Viviani et al., 1997; Actis-Grosso et al., 2001; or the coherence between trajectory and velocity profile: Papaxanthis et al., 1998; Pozzo et al., 2006).

In their general form, motor theories of perception claim that our perceptual systems is constrained (and supported) by the implicit knowledge with regard to the movements it is capable of producing. Hence, our hypothesis is that when visual input is partly missing, the brain would be more effective in SP reconstruction when supported by the memory template of a biological motion.

\section{EXPERIMENT 1 \\ MATERIALS AND METHODS \\ Participants}

Thirty-three participants voluntarily took part in this experiment (age range: 18-52 years old; mean age: 25.33 ; female: 13 ). They all had normal or corrected-to-normal vision and were all naïve as to the experimental purposes.

\section{Apparatus and stimuli}

The stimuli were displayed on a 22-inch color flat monitor $(1280 \times 1024$ pixels resolution, where a pixel can be considered a $0.37 \times 0.28 \mathrm{~mm}$ rectangle). All stimuli were handled and displayed using Psychophysics Toolbox extensions for Matlab (Brainard, 1997; Pelli, 1997). Each stimulus consisted in a white dot (10 pixels in width and 12 pixels in height, about $0.35^{\circ} \times 0.33^{\circ}$ of visual angle) moving upwards on black background. The dot moved within a presentation window of $1010 \times 680$ pixels centred on the screen; the size of this window was chosen so that the whole trajectory was far enough from the screen borders preventing participants from using reference points during the experiment running. Each trial was randomly displayed in a different position within the presentation window.

Two kinds of motion were displayed. For the first kind of motion (BIO) the dot moved upwards on the screen according to a normal biological rule, obtained as the average velocity profile of several upward arm pointing movements (arm angular displacement equal to $65^{\circ}$ ) previously recorded in frontal view (Papaxanthis et al., 1998). The motion displayed on the screen corresponded only to the last $60 \%$ of the total arm pointing movement.

For the non biological law of motion (NBIO) the stimulus followed a triangular shaped velocity profile, characterized by a linear acceleration in the first $60 \%$ of the trajectory, and a linear deceleration during the last $40 \%$ (see Figure 1). In this case as well the motion displayed on the screen corresponded only to the last $60 \%$ of the whole trajectory. The choice to use this particular law of motion was motivated by the fact that we wanted to make as easy as possible the reconstruction of SP in the NBIO condition, as described below.

Two reasons support the choice to show only upwards movements: (1) on the one hand, the choice of a vertical movement is due to the fact that only for vertical pointing movements the peak of velocity point (PVP) is not at the middle of the trajectory. Previous studies on pointing movements demonstrated that the velocity profiles of pointing movements are bell-shaped curves with different peak of velocity for horizontal, downwards and upwards movements, respectively. In particular the PVP is before the middle of the trajectory for upward movements (at 45\% from SP) and after the middle for downward movements (at 52\% from $\mathrm{SP})$, while it is at the middle of the trajectory for horizontal movements (Papaxanthis et al., 1998, 2003; Pozzo et al., 2006). PVP is a crucial type of information, being the cut point between the accelerated and decelerated phases of a biological motion. On the other hand (2) the choice to use only upwards movements is due to the fact that we wanted to avoid any confounding effect. It has been demonstrated that the CNS uses an internal model of gravity to supplement sensory information when estimating

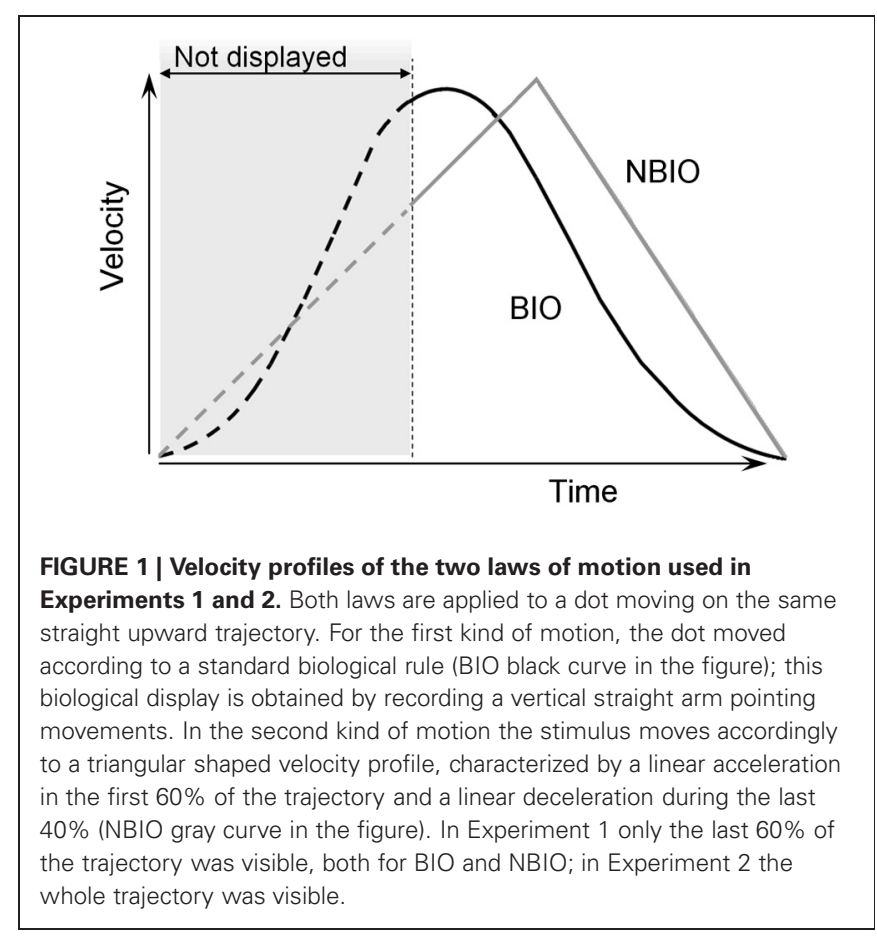


the interception point of a falling object (McIntyre et al., 2001; Senot et al., 2005), therefore it is possible that in this case as well knowledge about gravitational acceleration may be combined with visual-field information to provide an altered estimation of the SP for downwards movements (because the similitude between the two velocity profiles). A dark-gray horizontal line, 100 pixels in length and 2 pixels in height, was displayed to indicate the presence of a "covering area," and to provide a fixation point in the area where the dot was to appear. The line was located at the Appearing Point (AP) in both conditions. The moving dot appeared from the middle of the line, and then disappeared at the motion Ending Point (EP). The line was visible during the whole trial and disappeared only when the subject's response was given. For both conditions (BIO and NBIO) the whole trajectory from SP to EP (thus always including also the not displayed part) was $145 \mathrm{~mm}\left(13.6^{\circ}\right.$ of visual angle), and was traced by the disk in $2.07 \mathrm{~s}$; the mean velocity was $70.70 \mathrm{~mm} / \mathrm{s}$ (PVP $148.72 \mathrm{~mm} / \mathrm{s}$ ) in the BIO motion and $76.11 \mathrm{~mm} / \mathrm{s}$ in the NBIO motion (PVP $152.23 \mathrm{~mm} / \mathrm{s}$ ). Thus, BIO and NBIO motions differ also for the quality of information presented (see Figure 1): the visible acceleration phase (i.e., the phase between the AP and the PVP) in the $\mathrm{BIO}$ motion covers only the $5 \%$ of the visible trajectory, whereas in the NBIO condition it covers the $20 \%$. Furthermore, the instantaneous velocity at the AP is lower for the NBIO motion as compared to the $\mathrm{BIO}$ motion (respectively, $143.21 \mathrm{~mm} / \mathrm{s}$ for BIO motion and $101.49 \mathrm{~mm} / \mathrm{s}$ for NBIO motion). In this way all odds were against the possibility to detect any spurious effect: if participants would rely on a purely visual strategy to reconstruct the missing part of the trajectory, the visible part of the trajectory should determine SP localization. Thus, we should expect a better performance in SP localization for the NBIO condition, given both the lower instantaneous velocity at $\mathrm{AP}$ (slower velocities corresponds to smaller localization errors, e.g., Kerzel, 2002; ActisGrosso and Stucchi, 2003; Hubbard, 2005) and the greater amount of information available (i.e., longer visible acceleration phase).

\section{Procedure}

Each participant sat at a comfortable viewing distance from the screen (about $60 \mathrm{~cm}$ ) in a dimly light room. Participants were informed that the motion displayed on the screen corresponded to the motion of the finger extremity of an outstretched arm, performing an upward pointing movement in the sagittal plane. They were asked to make an informed guess as to the starting point of the movement and were encouraged to try to respond as accurately as possible.

A mouse click started the first trial, which began with a blank interval lasting $0.2-0.8 \mathrm{~s}$. The horizontal line was displayed; after a random delay of $0.8-1.6 \mathrm{~s}$ the target started its motion from the SP, becoming visible only after the AP (see Figure 2).

At the end of its motion the dot disappeared; a crosshair appeared in the center of the screen with a delay of $0.4 \mathrm{~s}$. Participants were asked to place the hair cross cursor where the motion started and to press the mouse button. The press of the button started the next trial.

Each condition had 16 replications, for a total of 32 trials presented in random order. The whole experiment lasted approximately $7 \mathrm{~min}$. The experimental protocol was carried

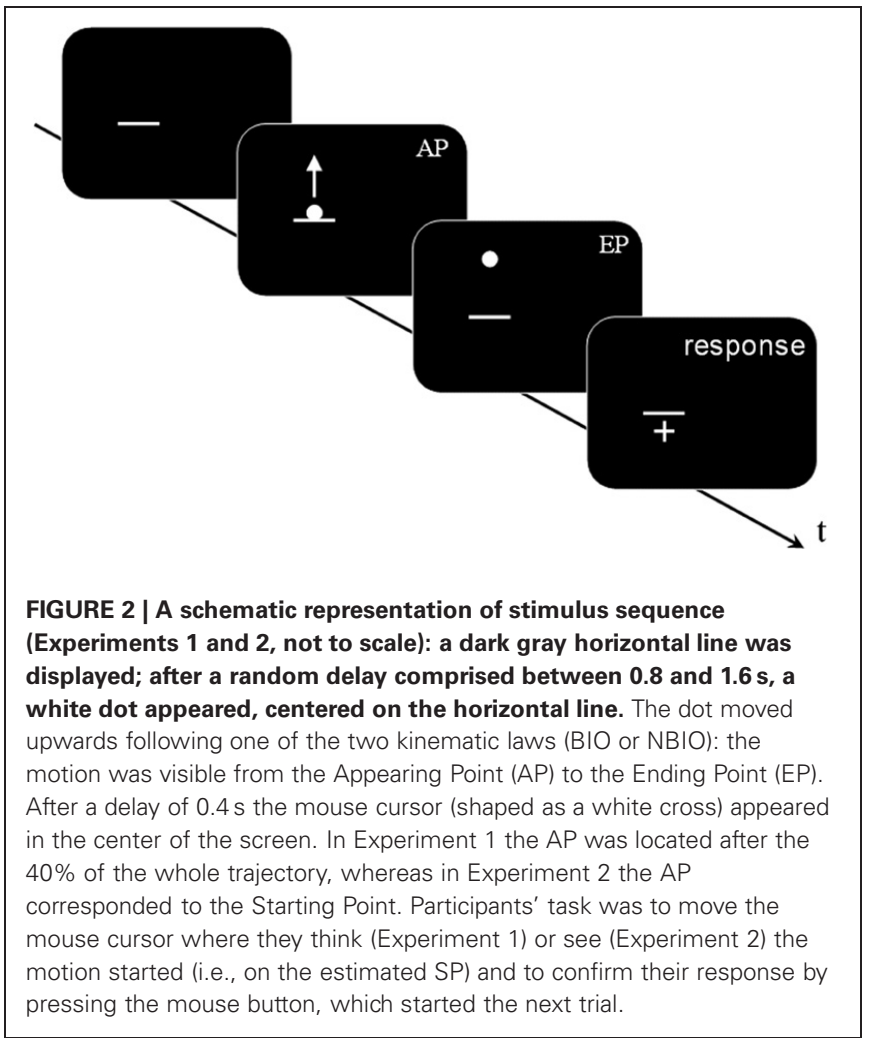

out in agreement with legal requirements and international norms (Declaration of Helsinki). All participants gave their written informed consent.

\section{Results and discussion}

Accuracy and precision of participants' estimations were given by Constant Error (CE), which corresponds to the inverse of the accuracy, and Variable Error (VE), which is the inverse of precision and is defined as the average of the individual standard deviations (SDs) calculated over the 16 replications carried out in each condition.

The mean CE was obtained by subtracting the coordinate of the response from the actual coordinate, thus obtaining a negative value for backward displacement and a positive value for forward displacement. Figure 3A presents the mean CE and its SD for each participant in each condition.

Table 1 summarizes the results for CE and VE; Horizontal $(\mathrm{H}$, along $\mathrm{x}$-axis) and Vertical ( $\mathrm{V}$, along $\mathrm{y}$-axis) projections of the errors are presented separately. Results show a perceived forward displacement of the SP for both BIO and NBIO motion conditions. Given that the contribution of the horizontal displacement was marginal ( $\leq 5 \%$ for both $\mathrm{BIO}$ and $\mathrm{NBIO}$ ) only the vertical component was analyzed. VEs were almost identical for the two motion conditions, indicating a good precision (i.e., consistency in making the same estimation several times) for both BIO and NBIO judgments.

An ANOVA on CEs with two within factors (Motion Condition and Repetition) revealed the significance of the main effect of Motion Condition $\left[F_{(1,32)}=4.3266\right.$, MS $=4416.45$, $p=0.046]$. However, data distribution significantly deviates 


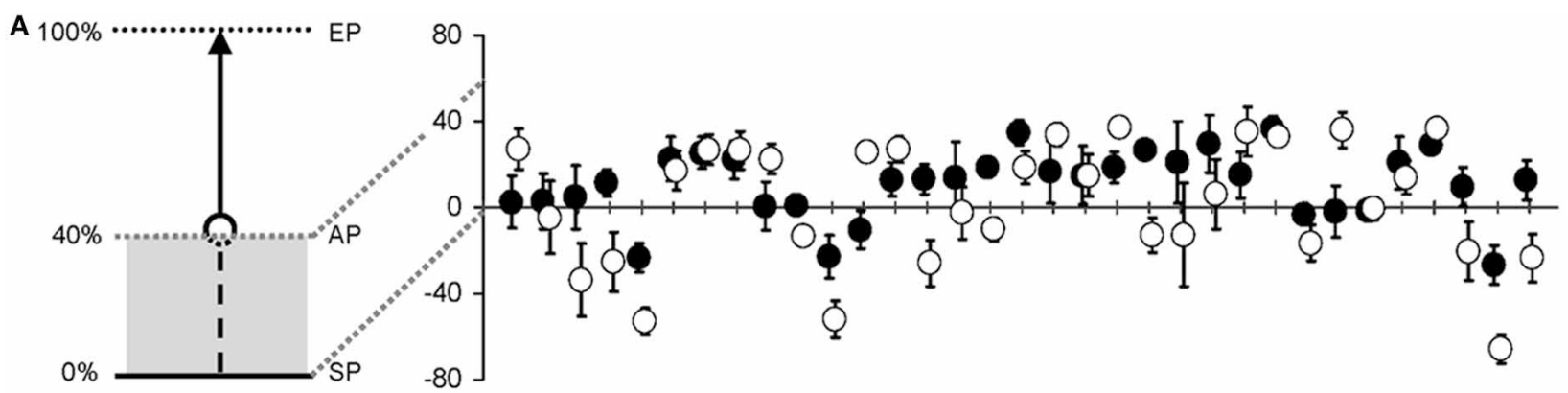

B

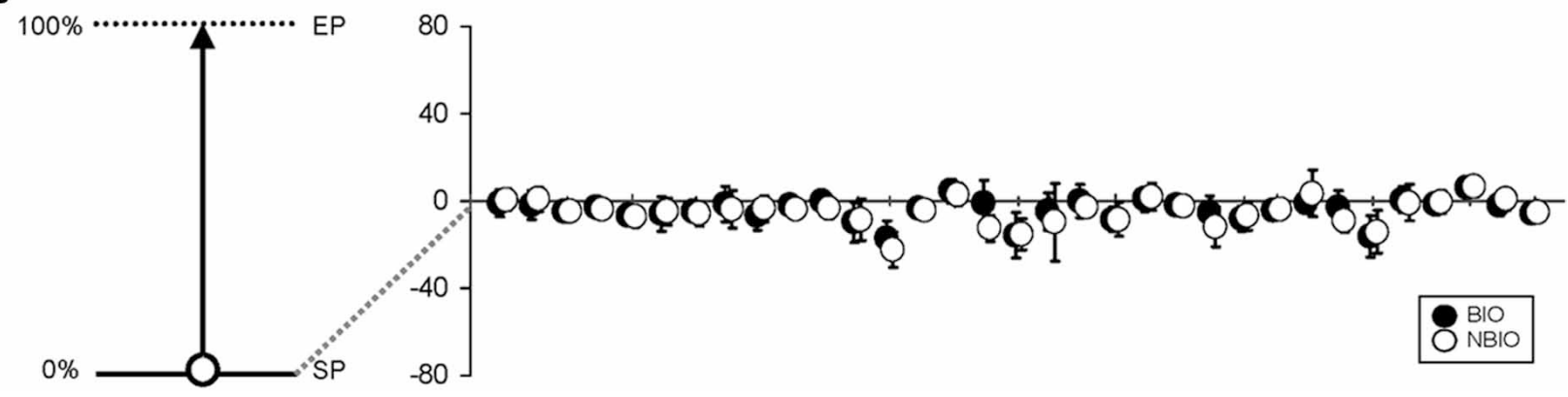

FIGURE 3 | (A) On the left, a schematic representation of the experimental set in Experiment 1: the beginning of the stimulus trajectory was hidden, and only the last $60 \%$ was displayed (along the trajectory: SP, Starting Point; $A P$, Appearing Point; EP, Ending Point). On the right, mean vertical error for the 33 participants (black dots for the Biological $(\mathrm{BIO})$ condition, white dots for the Non-Biological (NBIO) condition) for estimated SP (error bars for standard deviation). (B) On the left, a schematic representation of the experimental set in Experiment 2: the stimulus trajectory was completely visible. On the right, mean vertical error for the 33 participants [black dots for the Biological $(\mathrm{BIO})$ condition, white dots for the Non-Biological (NBIO) condition] for estimated SP (error bars for standard deviation).

Table 1 | Results of Experiment 1.

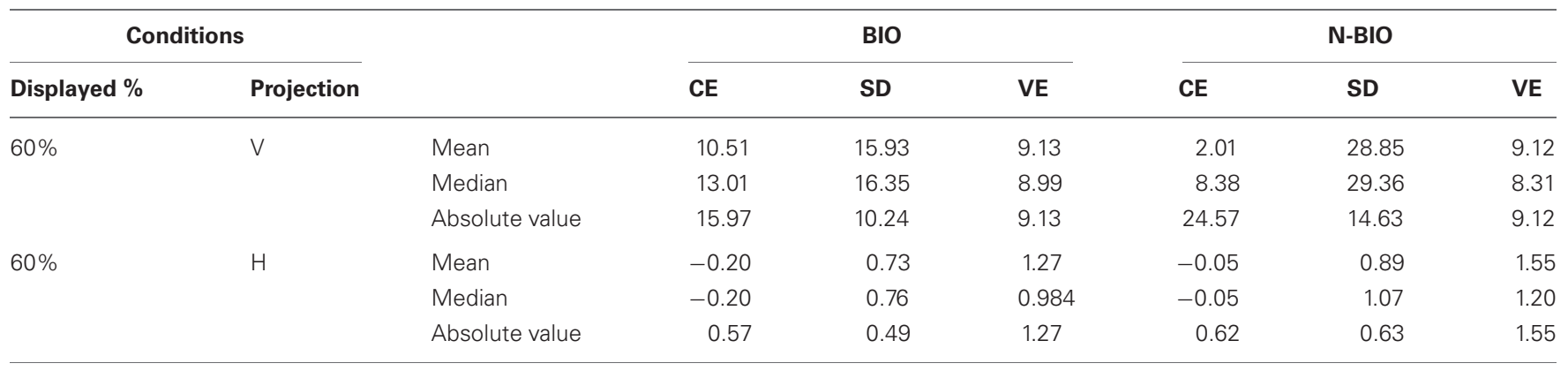

Constant Error (CE), Standard Deviation (SD), and Variable Error (VE) for the biological (BIO) and non-biological (NBIO) laws of motion, for both Horizontal (H) and Vertical (V) projection. In the table are presented the mean, median and absolute value errors. All values are in millimeter.

from normality (Kolmogorov-Smirnov test for normality distribution) suggesting at least some caution in considering mean values as central tendency indicators. For this reason median values were considered, as reported in Table 1. CE did not differ in the two motion conditions, whereas we found a significant difference in the variability: the SD in the NBIO condition is almost doubled $\left(F\right.$-test comparison of SDs $F_{(1,32)}=1.811$, $p<0.05)$. This difference in the variability could not be due to a worse performance in the NBIO condition, given that the VE values are almost identical in the two conditions; it is most likely due to a systematic tendency toward larger errors (both positive and negative): this information can easily be obtained by an analysis of the absolute value of estimations. The sign of CEs was thus changed to obtain positive values for both backward and forward displacements. An ANOVA on the absolute values of CEs, aimed at detecting systematic effects in the magnitude of CE, confirmed this hypothesis, revealing the significance of the main effect of Motion Condition $\left[F_{(1,32)}=11.3593, \mathrm{MS}=1484.4\right.$, $p=0.0019]$.

These results show the better efficacy in reconstructing the SP of a natural motion (i.e., following the BIO velocity profile) as compared to a motion violating the biological law: when the first part of a movement is hidden, all participants reconstruct the SP of a biological motion on the basis of the same general 
rule, whereas when the motion violates the biological kinematic law, participants use different strategies to reconstruct the SP, and show a tendency toward larger errors. However, this difference could be due to a better visual discrimination for the BIO motion as compared to the NBIO motion, resulting in an improved visual localization for the SP in BIO motion. A control experiment is thus necessary, where the full motion is displayed for both $\mathrm{BIO}$ and NBIO condition: if participants are more accurate in localizing the SP for the BIO motion when the full trajectory is displayed, we should conclude that the difference found in Experiment 1 could be due to a strategy based on visual mechanisms, not necessarily involving a specific internal model.

\section{EXPERIMENT 2 (CONTROL EXPERIMENT)}

A control experiment has been performed, where the full motion was displayed.

\section{MATERIALS AND METHODS}

Apparatus, stimuli, procedure, and general conditions were the same as in Experiment 1, with the following exceptions: the full trajectory ( $145 \mathrm{~mm}$ corresponding to $13.6^{\circ}$ of visual angle) was displayed, and the AP was located above the middle-point of the dark-gray horizontal line, at a random distance of 6-25 $\mathrm{mm}$. Participants were asked to place the hair cross cursor where the motion appeared and press the mouse button. Thirty-three participants voluntarily took part in this second experiment (age range: $17-38$ years old; mean age: 21.43 ; female: 12 ). They all had normal or corrected-to-normal vision and were all naïve as to the experimental purposes.

\section{Results}

Results showed a perceived backward displacement of the SP for both $\mathrm{BIO}$ and NBIO motion, consistent with previous results (e.g., Thornton, 2002; Hubbard and Motes, 2002; Actis-Grosso and Stucchi, 2003; Actis-Grosso et al., 2008). Figure 3B presents the mean CE and its SD for each participant and for each condition. A Two-Way ANOVA (two within factors: Motion Condition and Replication) shows a significant effect only for Replication $\left[F_{(15,480)}=1.7749, \mathrm{MS}=48.26, p=0.035\right]$; however, no trends are detectable in the dataset neither in the BIO nor in the NBIO condition.
For a better comparison with Experiment 1, median values and absolute values have been analyzed: no effect proved significant. In Table 2 the " $100 \%$ condition" summarizes the results (CE, SD, $\mathrm{VE})$ for the second experiment.

\section{GENERAL DISCUSSION}

In the present study we assessed the human's capacity to reconstruct the starting point of a moving dot. The starting point inference was more variable and incoherent when the observer reconstructed a non biological trajectory compared to a natural one. This advantage for biological motion is not present when the whole motion is displayed.

In Experiment 1 a biological and a non-biological motions are presented on the screen, both hidden for the first $40 \%$ of the trajectory. We chose to compare a natural motion with a specific non-biological profile, which maximizes the information for the reconstruction of the SP. Despite the stimulation length is the same, the two conditions are characterized by a different quality of information presented to the observers (see Figure 1): the visible accelerating phase (i.e., the phase between the AP and the $\mathrm{PVP}$ ) in the $\mathrm{BIO}$ condition lasts only the $5 \%$ of the whole trajectory, whereas in the NBIO condition it lasts the $20 \%$ of the whole trajectory. This, associated to a lower instantaneous velocity at the AP for the NBIO condition, allows one to expect a better performance in SP localization for the non-biological kinematics. In the case of purely visual extrapolation, the visible part of the trajectory should determine SP estimation. Consequently, the display presenting a longer visible acceleration phase and a lower velocity at the AP would lead to greater precision and accuracy in starting point estimation than the display with faster and shorter visible acceleration phase. A more conservative hypothesis would predict no difference between $\mathrm{BIO}$ and NBIO motion. The results of the first experiment instead show a better efficacy in reconstructing the SP of a natural motion (i.e., following the BIO velocity profile) as compared to a motion violating the biological law: with BIO motion all participants reconstruct the SP on the basis of the same general rule, whereas when the motion violates the biological kinematic law, participants use different strategies to reconstruct the SP, and show a tendency toward larger errors.

To clarify the role of the local visual mechanisms and the internal representation to support the localization task, we performed

Table 2 | Results of Experiment 2.

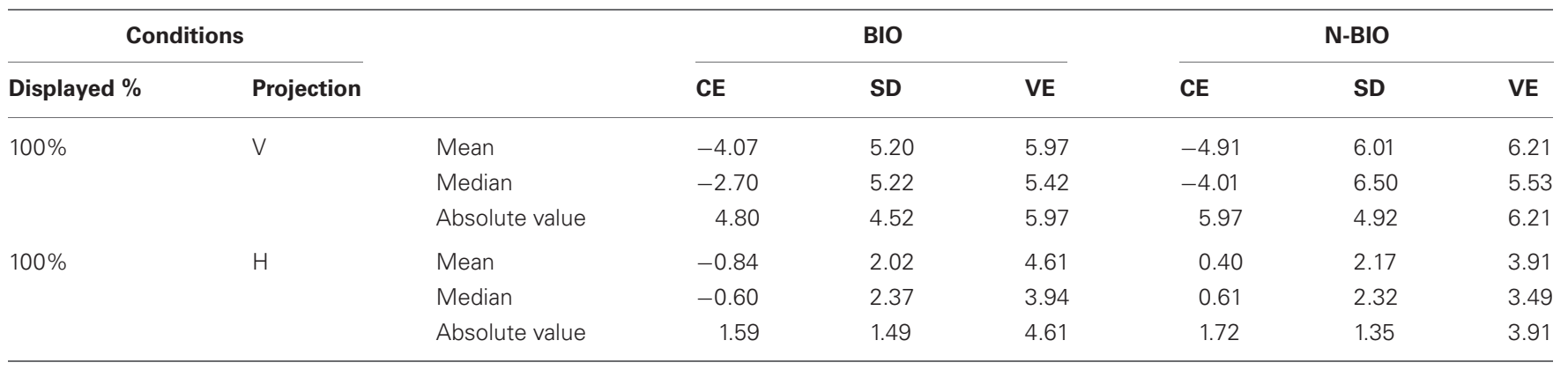

Constant Error (CE), Standard Deviation (SD), and Variable Error (VE) for the biological (BIO) and non-biological (NBIO) laws of motion, for both Horizontal (H) and Vertical (V) projection. In the table are presented the mean, median, and absolute value errors. All values are in millimeter. 
a second experiment wherein we compared the biological and the non-biological law of motion, now displaying the whole trajectories.

Previous studies investigating the localization of perceived SP highlighted the presence of a systematic error. In those studies a constant velocity stimulus was presented in horizontal or in vertical direction; they found a systematic bias in the direction of motion (e.g., Müsseler and Aschersleben, 1998) or in the opposite direction (e.g., Kerzel, 2002; Thornton, 2002; Actis-Grosso and Stucchi, 2003). Furthermore, in a study where velocity was modulated in the first, central, and last part of a horizontal or vertical motion path (Actis-Grosso et al., 2008), accelerations and decelerations had no effect on SP estimation, suggesting that a local visual mechanism is responsible for SP localization.

The results of Experiment 2 are in line with this view: we found an error in the direction opposite to motion and no difference between the BIO and NBIO conditions, supporting the idea that SP localization is based on a purely visual perception mechanisms and local visual measurements, since the estimation was not found sensitive to the kinematic of the display. This insensitivity was already found in Pozzo et al. (2006) where participants were not able to explicitly recognize biological and non-biological motion and no difference was found between BIO and NBIO motion for the perception of the final position when the full motion was displayed.

In contrast, it is evident that visual perception alone cannot explain our capacity to reconstruct the past. The advantage found for biological motion in SP inference (and not in perceptual judgment) suggests that when visual information is missing the starting point estimation would rely on a stored stereotyped kinematic pattern characterizing the displayed upward arm movement. This result should be considered as a first step toward a full understanding of human ability in reconstructing the SP of a natural motion (i.e., in recovering the "past" trajectory): we are actually working on

\section{REFERENCES}

Actis-Grosso, R., Bastianelli, A., and Stucchi, N. (2008). Direction of perceptual displacement of a moving target's starting and vanishing points: the key role of velocity. Jpn. Psychol. Res. 50, 253-263.

Actis-Grosso, R., de'Sperati, C., Stucchi, N., and Viviani, P. (2001). "Visual extrapolation of biological motion," in Fechner Day 2001. Proceedings of the Seventeenth Annual Meeting of the International Society for Psychophysics, eds E. Sommerfeld, R. Kompass and T. Lachmann (Lengerich, Germany: Pabst Science Publishers), 261-266.

Actis-Grosso, R., and Stucchi, N. (2003). Shifting the start: backward mislocation of the initial position of a motion. J. Exp. Psychol. Hum. Percept. Perform. 29, 675-691.

Brainard, D. H. (1997). The psychophysics toolbox. Spat. Vis. 10, 433-436.
Hubbard, T. L. (2005). Representational momentum and related displacements in spatial memory: a review of the findings. Psychon. Bull. Rev. 12, 822-851.

Hubbard, T. L., and Motes, M. A. (2002). Does representational momentum reflect a distortion of the length or the endpoint of a trajectory? Cognition 82, B89-B99.

Johansson, G. (1973). Visual perception of biological motion and a model for its analysis. Percept. Psychophys. $14,201-211$.

Jordan, M. I., and Rumelhart, D. E. (1992). Forward models - supervised learning with a distal teacher. Cogn. Sci. 16, 307-354.

Kerzel, D. (2002). Different localization of motion onset with pointing and relative judgements. Exp. Brain Res. $145,340-350$.

Kilner, J. M., Friston, K. J., and Frith, C. D. (2007). Predictive

a larger selection of non-biological profiles and amounts of occlusion.

The present study complements the previous study by Pozzo et al. (2006), where accuracy increased for the estimation of the final position of a biological motion. In the present study we found an advantage for the estimation of the starting position as well, supporting the idea that as soon as the target appears the cortical motor area would generate an internal representation of reaching movement in the upward direction. The visual input would resonate with top down input only if the kinematic of the two inputs are sufficiently similar. Pozzo et al. (2006) suggested that, from the simulated movement, the forward model that predicts the sensory consequence of the movement could be used to compensate the lack of visual input due to occlusion. We suggest that such internal model could be used also to reconstruct the past part of a motion: when the visual input and the stored kinematic template match, the SP is traced back on the basis of this memory template; in contrast, when the kinematics of the two inputs are different, a purely visual strategy is used. This demonstrates that a low-level visual attribute of motor activity such as the kinematic features of a simple dot is used by the visual system to categorize movement as biological or non-biological and thus reaches (or not) the motor system of the observer.

During daily life activity actions are performed toward a goal (e.g., an object to reach) that concentrates a significant part of attentional resources, in contrast to the initial conditions, a non-essential parameter for the survival, but a relevant dataset supporting the comprehension of the present and the foreseen toward the future. Even if compatible with the present result suggesting inference process on the basis of motor competencies, additional empirical evidences should confirm such hypothesis.

\section{ACKNOWLEDGMENTS}

This research was supported by the French ANR (Simaction, projet Tecsan).

coding: an account of the mirror neuron system. Cogn. Process. 8, 159-166.

Lacquaniti, F., Terzuolo, C., and Viviani, P. (1983). The law relating the kinematic and figural aspects of drawing movements. Acta Psychol. (Amst.) 54, 115-130.

McIntyre, J., Zago, M., Berthoz, A., and Lacquaniti, F. (2001). Does the brain model Newton's laws? Nat. Neurosci. 4, 693-694.

Miall, R. C., and Wolpert, D. M. (1996). Forward models for physiological motor control. Neural Netw. 9, 1265-1279.

Müsseler, J., and Aschersleben, G. (1998). Localizing the first position of a moving stimulus: the Frohlich effect and an attention-shifting explanation. Percept. Psychophys. 60, 683-695.

Nijhawan, R. (1994). Motion extrapolation in catching. Nature 370, 256-257.
Papaxanthis, C., Dubost, V., and Pozzo, T. (2003). Similar planning strategies for whole-body and arm movements performed in the sagittal plane. Neuroscience 117, 779-783.

Papaxanthis, C., Pozzo, T., and Stapley, P. (1998). Effects of movement direction upon kinematic characteristics of vertical arm pointing movements in man. Neurosci. Lett. 253 103-106.

Pelli, D. G. (1997). The VideoToolbox software for visual psychophysics: transforming numbers into movies. Spat. Vis. 10, 437-442.

Pozzo, T., Papaxanthis, C., Petit, J. L., Schweighofer, N., and Stucchi, N. (2006). Kinematic features of movement tunes perception and action coupling. Behav. Brain Res. 169, 75-82.

Senot, P., Zago, M., Lacquaniti, F., and McIntyre, J. (2005). Anticipating the effects of gravity when intercepting 
moving objects: differentiating up and down based on nonvisual cues. J. Neurophysiol. 94, 4471-4480.

Thornton, I. M. (2002). The onset repulsion effect. Spat. Vis. 15, 219-243.

Viviani, P., Baud-Bovy, G., and Redolfi, M. (1997). Perceiving and tracking kinesthetic stimuli: further evidence of motor-perceptual interactions. J. Exp. Psychol. Hum. Percept. Perform. 23, 1232-1252.
Viviani, P., and Stucchi, N. (1989). The effect of movement velocity on form perception: geometric illusions in dynamic displays. Percept. Psychophys. 46, 266-274.

Viviani, P., and Stucchi, N. (1992). Biological movements look uniform: evidence of motorperceptual interactions. J. Exp. Psychol. Hum. Percept. Perform. 18, 603-623.

Wolpert, D. M., and Kawato, M. (1998). Multiple paired forward and inverse models for motor control. Neural Netw. 11, 1317-1329.

Conflict of Interest Statement: The authors declare that the research was conducted in the absence of any commercial or financial relationships that could be construed as a potential conflict of interest.

Received: 20 March 2012; accepted: 29 May 2012; published online: 14 June 2012
Citation: Carlini A, Actis-Grosso $R$, Stucchi $N$ and Pozzo $T$ (2012) Forward to the past. Front. Hum. Neurosci. 6:174. doi: 10.3389/fnhum.2012.00174

Copyright (c) 2012 Carlini, ActisGrosso, Stucchi and Pozzo. This is an open-access article distributed under the terms of the Creative Commons Attribution Non Commercial License, which permits non-commercial use, distribution, and reproduction in other forums, provided the original authors and source are credited. 